\title{
Immunogenicity Specimen Assessments Subcategory
}

National Cancer Institute

\section{Source}

National Cancer Institute. Immunogenicity Specimen Assessments Subcategory. NCI

Thesaurus. Code C117558.

A subdivision of immunogenicity specimen assessments. 